$\mathrm{KBC}-40653$

\title{
Integrated Water \\ Treatment System \\ Performance Evaluation
}

Prepared for the U.S. Department of Energy

Assistant Secretary for Environmental Management

Contractor for the U.S. Department of Energy

under Contract DE-AC06-08RL14788

CH2MHILL

Plateau Remediation Company

P.O. Box 1600

Richland, Washington 99352 
KBC-40653

Revision 0

EDC \#: PRC-EDC-09-40654

\section{Integrated Water Treatment System Performance Evaluation}

Document Type: TR

Program/Project: STP

R. A. Sexton

W. E. Meeuwsen

CH2M HILL Plateau Remediation Company

Date Published

March 2009

Prepared for the U.S. Department of Energy

Assistant Secretary for Environmental Management

Contractor for the U.S. Department of Energy

under Contract DE-AC06-08RL14788

CHIMHILL

Plateau Remediation Company

P.O. Box 1600

Richland, Washington

$\frac{\text { 2. Si, Racalal }}{\text { Release Approval }}$

$\frac{03 / 12 / 09}{0 a t e}$

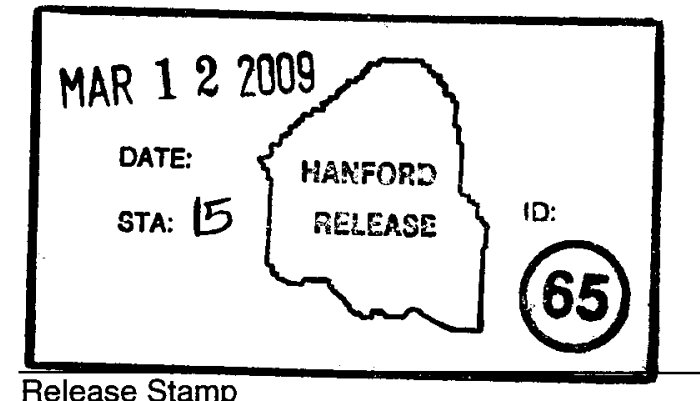

Release Stamp 
KBC-40653

Revision 0

TRADEMARK DISCLAIMER

Reference herein to any specific commercial product, process,

or service by trade name, trademark, manufacturer, or

otherwise, does not necessarily constitute or imply its

endorsement, recommendation, or favoring by the United

States Government or any agency thereof or its contractors or subcontractors.

This report has been reproduced from the best available copy.

Printed in the United States of America

Total Pages:

16 


\section{KBC-40653, Rev. 0 \\ Integrated Water Treatment System Performance Evaluation Contents}

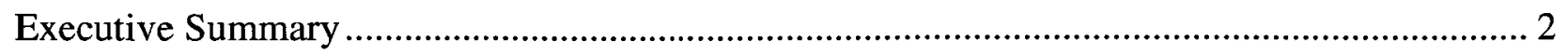

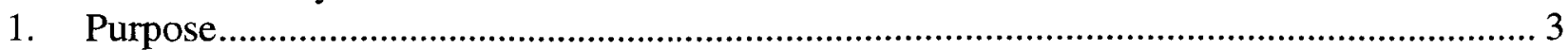

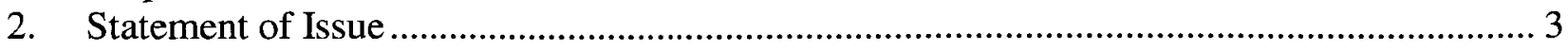

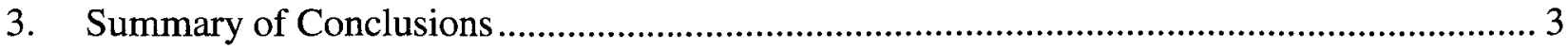

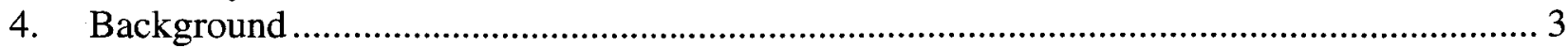

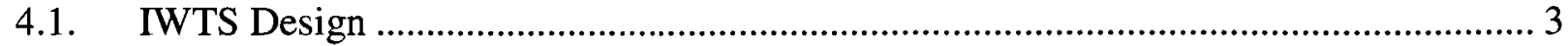

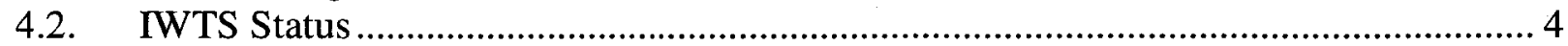

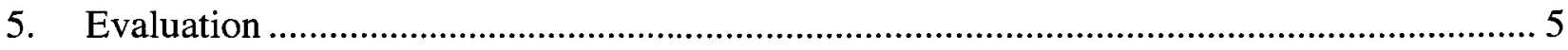

5.1. Hydraulic Model Compared to System Data ........................................................... 5

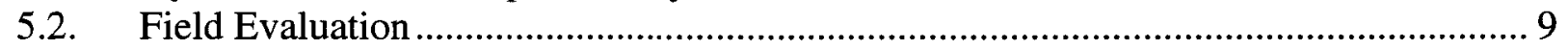

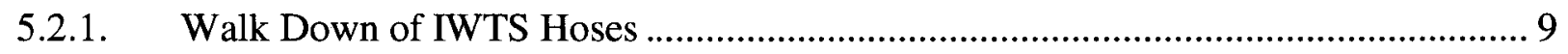

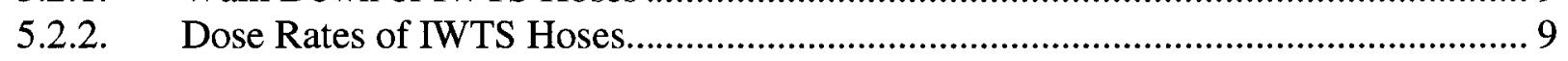

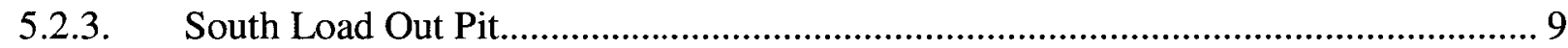

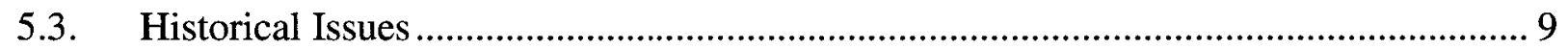

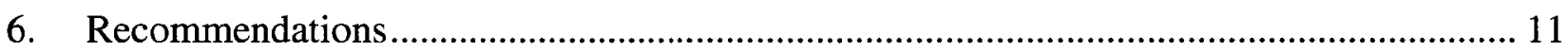

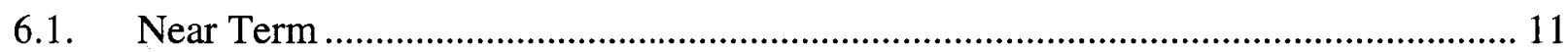

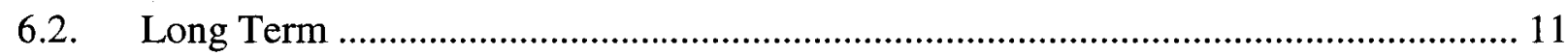

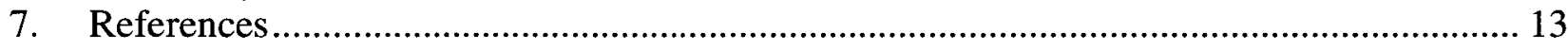




\section{Executive Summary}

This document describes the results of an evaluation of the current Integrated Water Treatment System (IWTS) operation against design performance and offers recommended short term and long term actions to sustain IWTS performance.

The KW IWTS was designed to treat basin water and maintain basin clarity during fuel retrieval, washing, and packaging activities in the KW Basin. The original design was based on a mission that was limited to handling of KW Basin fuel.

The use of the IWTS was extended by the decision to transfer KE fuel to KW to be cleaned and packaged using KW systems. The use was further extended for the packaging of two more Multi-Canister Overpacks (MCOs) containing legacy fuel and scrap. Planning is now in place to clean and package Knock Out Pot (KOP) Material in MCOs using these same systems. Some washing of KOP material in the Primary Cleaning Machine (PCM) is currently being done to remove material that is too small or too large to be included in the KOP Material stream. These plans will require that the IWTS remain operational through a campaign of as many as 30 additional MCOs, and has an estimated completion date in 2012.

Recent operation of the IWTS during washing of canisters of KOP Material has been impacted by low pressure readings at the inlet of the P4 Booster Pump. The system provides a low pressure alarm at $10 \mathrm{psig}$, and low-low pressure interlock at 5 psig. The response to these low readings has been to lower total system flow to between 301 and $315 \mathrm{gpm}$. In addition, the IWTS operator has been required to operate the system in manual mode and make frequent adjustments to the P4 booster pump speed during PCM washes. The preferred mode of operation is to establish a setpoint of $317 \mathrm{gpm}$ for the P4 pump speed and run IWTS in semi-automatic mode.

Based on hydraulic modeling compared to field data presented in this report, the low P4 inlet pressure is attributed to restrictions in the 2" KOP inlet hose and in the KOP itself. The flow velocity in these areas should not allow accumulations of sludge particles. The restrictions are potentially attributed to the nesting of wire-like material that is small enough in two dimensions to pass through the PCM.

The data does not indicate that IWTS performance has been degraded by pump wear or blockage, by restrictions in the 3 " or 4 " IWTS hoses, by accumulation of material in the settler, by leakage, or by failure or degradation of other system components.

Near term recommendations of this document focus on replacing the KOP inlet hose and removing any accumulated material in the KOP inlet. Long term recommendations focus on monitoring system performance, stocking appropriate spare parts and performing a more comprehensive evaluation of all MCO handling systems. 


\section{Purpose}

This document describes the results of an evaluation of the current IWTS operation against design performance and a determination of short term and long term actions recommended to sustain IWTS performance.

\section{Statement of Issue}

Recent operation of the IWTS during washing of canisters of KOP Material has been impacted by low pressure readings at the inlet of the P4 Booster Pump. The system provides a low pressure alarm at $10 \mathrm{psig}$, and low-low pressure system shutdown interlock at $5 \mathrm{psig}$. The response to these low readings has been to lower total system flow to between 301 and $315 \mathrm{gpm}$. In addition, the IWTS operator has been required to operate the system in manual mode and make frequent adjustments to the P4 booster pump speed during PCM washes. The preferred mode of operation is to establish a setpoint of $317 \mathrm{gpm}$ for the P4 pump speed and run IWTS in semi-automatic mode.

\section{Summary of Conclusions}

Based on modeling discussed herein, the low P4 inlet pressure is attributed to restrictions in the 2" KOP inlet hose and in the KOP itself. The flow velocity in these areas should not allow accumulations of sludge particles. The restrictions are potentially attributed to the nesting of wire-like material that is small enough in two dimensions to pass through the PCM.

The data does not indicate that IWTS performance has been degraded by pump wear or blockage, by restrictions in the 3" or 4" IWTS hoses, by accumulation of material in the settler, by leakage, or by failure or degradation of other system components.

Near term recommendations of this document focus on replacing the KOP inlet hose and removing any accumulated material in the KOP inlet. Long term recommendations focus on monitoring system performance, stocking appropriate spare parts and performing a more comprehensive evaluation of all MCO handling systems.

\section{Background}

\subsection{IWTS Design}

The KW IWTS was designed to treat basin water and maintain basin clarity during fuel retrieval, washing, and packaging activities in the KW Basin. The original design was based on a mission that was limited to handling of KW Basin fuel.

Figure 1 is a Process Flow Diagram of the IWTS system. Pumps P1, P2, and P3 were designed to draw from canister decapping, the PCM, and the process table, respectively, for a combined flow of $320 \mathrm{gpm}$. This combined stream is routed through the KOP, the settler, the garnet filters, and the ion exchange modules.

System Design Description for the KW Basin Integrated Water Treatment System, SNF-4449, Rev. 0, April, 2000, provides a detailed description of the IWTS design. The system was later modified to remove the 500 micron screens internal to the KOP and install 30 mesh strainers downstream of the KOP. 


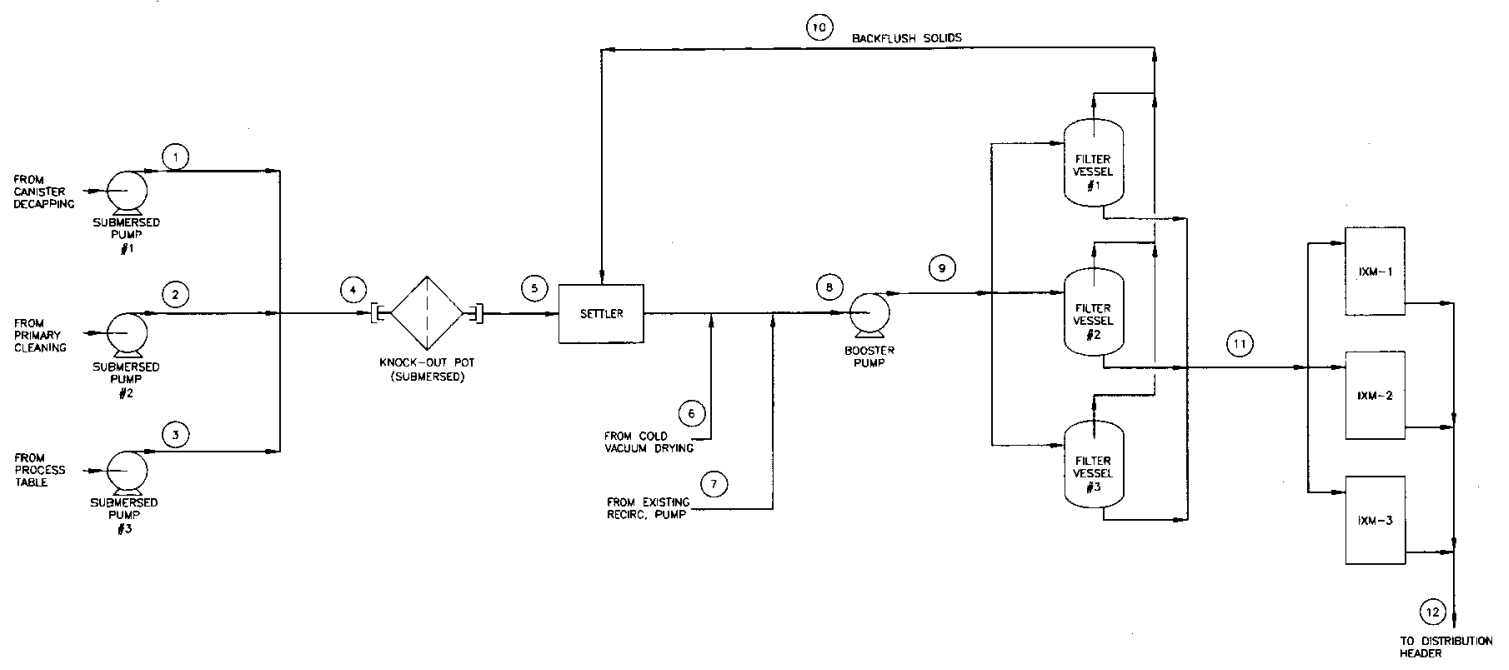

\section{Figure 1 - IWTS Process Flow Diagram}

\subsection{IWTS Status}

The use of the IWTS was extended by the decision to transfer KE fuel to KW to be cleaned and packaged using KW systems. The use was further extended for the packaging of two more MCOs containing legacy fuel and scrap. Planning is now in place to clean and package KOP Material in MCOs using these same systems. Some washing of KOP material in the PCM is currently being done to remove material that is too small or too large to be included in the KOP Material stream. Planned use of the IWTS includes finishing this wash cycle, performing a second wash cycle, density separation and washing the resultant scrap. This will require that the IWTS remain operational through a campaign of as many as 30 additional MCOs, and has an estimated completion date in 2012. 


\section{Evaluation}

\subsection{Hydraulic Model Compared to System Data}

\section{Analysis Method:}

Field recorded data ranging from 2002 (pre IWTS strainer installation) to present day (including P-4 low inlet pressure events), was reverse engineered through hydraulic modeling. The calculated pressures and flow rates at key points in the IWTS were compared to recorded data from the field. Manual iterations were performed using the model until the pressure profiles of the hydraulic model and field matched.

Modeled upset conditions included leaks, throttled inlets and/or outlets of pumps in various combinations, modified pump curves simulating worn impellers, modified pump speeds simulating solids wedged in the pump, throttled various locations throughout the system, and increased or decreased resistance curves for KOPs, strainers, filter vessels, and IXMs.

\section{Key Findings:}

Two unique conditions had to be added to the bench mark model cases to reproduce the current P-4 low inlet pressure field readings. Both have to occur for the model to match the field. Other scenarios were run but did not reproduce the field readings (see "Scenarios Run" below). The conditions that were added consist of:

Condition 1: $\quad$ KOP Inlet hose differential pressure has to be increased to 23 psi to cause low pressure events (historically $8.5 \mathrm{psi}$ ).

Condition 2: $\quad$ KOP vessel differential pressure has to be increased to almost 30 psi to cause low pressure events (historical values are 19 psi for KOP \#3, 22 psi for KOP \#10 and 11 psi for KOP \#11).

Condition 1 is required to reproduce the current field readings at the inlet of the KOP vessel as recorded by pressure instrument PIT-206-05. Condition 2 is required to reproduce the current field readings at the inlet of the IWTS strainers as recorded on pressure instrument PIT-206-06. Once these two conditions were added to the model, the system pressures and flow rates of the remaining IWTS matched the current field recorded readings.

The modeling indicates that velocities in these areas are high enough to preclude accumulation of sludge particles. This suggests a possible nesting of wire or similar material that could have passed through the PCM.

\section{Scenarios Run:}

After bench marking the model based on 2002 (pre IWTS strainer install) and 2003 (post IWTS strainer) data, approximately 50 scenarios were performed in an attempt to reproduce the low P-4 inlet pressure events. A condensed list of conditions reviewed is as follows:

- Leak from system ranging from pin hole to $30 \mathrm{gpm}$. Leak location placed at P-2 discharge, strainer inlet, KOP and P-4 inlet both single and in combination:

- Conclusion $\rightarrow$ All failed to reproduce field recorded data.

- Throttled inlet and/or outlet of all pumps in various combinations: 
KBC-40653, Rev. 0

- Conclusion $\rightarrow$ All failed to reproduce field recorded data.

- Modified pump curves to simulate worn impellers:

- Conclusion $\rightarrow$ All failed to reproduce field recorded data.

- Modified speeds of pumps to simulate solids wedged between impeller and casing (mimics a reduced speed of pump) or incorrect VFD display on P-4:

- Conclusion $\rightarrow$ All failed to reproduce field recorded data.

- Throttled various locations throughout the system (e.g., mimic partial blockages due to solids, tight hose bends, hose kinks/crushes, etc.):

- Conclusion $->$ Most failed to reproduce field recorded data with the exception of the KOP Inlet Hose increased loss.

- Increased and decreased resistance curves for KOP, Strainers, Filter Vessels and IXMs.

- Conclusion $->$ Most failed to reproduce field recorded data with the exception of the KOP vessel.

IWTS Strainers, Settlers, Filter Vessels and IXMs appear to be operating consistently with past operating data. No significant increase or decrease in pressure drop was found nor any off normal behavior observed. Increasing the percentage of blockage (i.e., percent full) of the settlers had insignificant impact on the pressure profile and flow rates of the IWTS. Even when the settlers were assumed to be essentially full, the resulting pressure profile does not match field records, specifically the pressure at the KOP inlet, strainer inlet, strainer outlet and P-4 inlet.

\section{Critical Velocity:}

The increase in pressure drop on KOP inlet hose not supported by critical velocity calculations (i.e., the hose should self clean). The methodology for slurry flow of compound systems prepared by Dr. Edward Wasp was used for critical velocity and pressure drop prediction:

- At solids volume concentration of $10 \%$, critical velocity $\sim 11.8$ fps

- At solids volume concentration of $2 \%$, critical velocity $\sim 7.5 \mathrm{fps}$.

- Velocity in the 2 inch KOP Inlet Hose typically 32 fps.

This suggests that the additional loss is due to partial blockage from trapped debris (long, entangled material such as wire, springs) in KOP the inlet hose and KOP vessel inlet pipe.

\section{Solids Transport:}

There is insufficient data on the time and characteristics of solids transport to evaluate their impact on IWTS performance. However the results suggest that the effects of transferred solids are small and not enough to produce the observed pressure profile.

\section{Model Results:}

Table 1 summarizes the results of the modeling, compared to field data. Runs 5 and 6 in the table predict expected results from replacing the KOP inlet hose, and removing the pressure loss in the KOP. 
Table 1 - IWTS Model Results

\begin{tabular}{|c|c|c|c|c|c|c|c|c|c|c|c|c|c|c|c|c|c|}
\hline Condition/Era & $\begin{array}{c}\text { Special } \\
\text { Condition(s) }\end{array}$ & $\begin{array}{c}\text { P-1 Flow } \\
\text { (gpm) }\end{array}$ & $\begin{array}{c}\text { P-2 Flow } \\
\text { (gpm) }\end{array}$ & $\begin{array}{c}\text { P-3 Flow } \\
\text { (gpm) }\end{array}$ & $\begin{array}{c}\text { P-4 Flow } \\
(\mathrm{gpm})\end{array}$ & $\begin{array}{c}\text { P-4 Inlet } \\
\text { Pressure } \\
\text { (psig) }\end{array}$ & $\begin{array}{c}\text { P-4 Outlet } \\
\text { Pressure } \\
\text { (psig) }\end{array}$ & $\begin{array}{c}\text { KOP Inlet } \\
\text { Pressure } \\
\text { (psig) }\end{array}$ & \begin{tabular}{|c|} 
Strainer \\
Inlet \\
Pressure \\
(psig) \\
\end{tabular} & \begin{tabular}{|c|} 
Strainer \\
Outlet \\
Pressure \\
(psig) \\
\end{tabular} & \begin{tabular}{|c|} 
Settler \\
Inlet \\
Pressure \\
(psig) \\
\end{tabular} & \begin{tabular}{|c|} 
Settler \\
Outlet \\
Pressure \\
(psig) \\
\end{tabular} & $\begin{array}{c}F V-1 \Delta p \\
\text { (psig) }\end{array}$ & $\begin{array}{c}F V-2 \Delta p \\
\text { (psig) }\end{array}$ & $\begin{array}{c}F V-3 \Delta p \\
\text { (psig) }\end{array}$ & $\begin{array}{c}\text { IXM Inlet } \\
\text { Pressure } \\
\text { (psig) }\end{array}$ & $\begin{array}{c}\text { IXM Outlet } \\
\text { Pressure } \\
\text { (psig) }\end{array}$ \\
\hline \multicolumn{18}{|l|}{ Pre Strainer Operations } \\
\hline Previous Calc (SNF-6840, 2000) & $\mathrm{A}$ & 83.6 & 83.8 & 152.6 & 320 & 43 & 99.4 & 103.8 & $\mathrm{NA}$ & $\overline{\mathrm{NA}}$ & 59.33 & 57.16 & 20.3 & 20.45 & 20.45 & 64.4 & 33.31 \\
\hline Field Data (3/29/2002 midnight - $6: 00 \mathrm{am}) \mathrm{KOP} \# 3$ & & 83.9 & 84.3 & 142.8 & 312.2 & 37.8 & 63 & $\overline{\mathrm{NA}}$ & $\overline{N A}$ & $\overline{\mathrm{NA}}$ & NA & $\overline{\mathrm{NA}}$ & 13.3 & 16.6 & $\overline{N A}$ & 44.2 & 32.7 \\
\hline Field Data $(4 / 1 / 2002$ noon $-6: 00 \mathrm{pm}) \mathrm{KOP} \# 3$ & & 85.7 & 88.5 & 142.8 & 317 & 34.5 & 64.4 & NA & NA & NA & NA & NA & 14.9 & 18.2 & NA & 43.8 & 32.2 \\
\hline Run \#1 Bench Marking, This calc - 2009 & $\mathrm{~B}$ & 87.7 & 87.7 & $\overline{141.7}$ & 317.3 & 36.9 & 67.4 & 96 & NA & NA & 49.9 & 51 & 13.13 & 13.02 & 12.56 & 43.3 & 31.2 \\
\hline Field Data $(7 / 15 / 2003$ noon - $6: 00 \mathrm{pm})$ KOP \#10 & & 90 & 99.5 & 128.5 & 317.8 & 27.5 & 60.5 & $\overline{N A}$ & $\mathrm{NA}$ & NA & $\mathrm{NA}$ & $\overline{N A}$ & 14.7 & 17.9 & $\overline{\mathrm{NA}}$ & 39.8 & 32.7 \\
\hline Run \#2 Bench Marking, This calc - 2009 & $\mathrm{C}$ & 93.4 & 95.8 & 128.2 & 317.81 & 29.5 & 61.9 & 92.9 & 70.3 & 66.8 & 42.5 & 43.7 & 13.1 & 13.5 & 12.6 & 37.8 & 27.3 \\
\hline \multirow{2}{*}{\multicolumn{18}{|c|}{ Post Strainer Operations }} \\
\hline & & & & & & & & & & & & & & & & & \\
\hline Field Data (8/12/2003 midnight - 8/12/2003 noon) KOP \#11 & $\mathrm{D}$ & 124.6 & 76.6 & 114.9 & 316 & 40.7 & 78.8 & 82.8 & 73 & 68.5 & $\mathrm{NA}$ & $\mathrm{NA}$ & 33.6 & 35.6 & $\mathrm{NA}$ & 40.5 & 33.5 \\
\hline Run \#3 Bench Marking, This calc - 2009 & & 119.3 & 73.9 & 122.7 & 316 & 32.2 & 80.2 & 83.2 & 73.1 & 72.5 & 45 & 46.2 & 30.4 & 30.2 & 29.75 & 39 & 28.6 \\
\hline \multicolumn{18}{|l|}{ Recent Events } \\
\hline Field Data $(1 / 15 / 093: 00-4: 00 \mathrm{pm})$ KOP \#12 & & 83.6 & 68.1 & $\overline{163.8}$ & 315.6 & 8.9 & 57.4 & 80.8 & 50.6 & 47.2 & $\overline{\mathrm{NA}}$ & $\overline{N A}$ & 15.85 & $50 ?$ & $\overline{\mathrm{NA}}$ & 343 & 21.5 \\
\hline Field Data $(1 / 16 / 093: 00$ - 4:00 pm) KOP \#12 & & 80.5 & 61.4 & 165.9 & 307.8 & 10.8 & 67.8 & 82.2 & 51 & 47.7 & $\mathrm{NA}$ & NA & 26.4 & $50 ?$ & NA & 33.4 & 21 \\
\hline Run \#4, This calc - 2009 & $\mathrm{E}$ & 81.9 & 68.4 & 157.7 & 308 & 11.7 & 55 & 83.5 & 54.2 & 50.3 & 24.2 & 25.4 & 13.1 & 12 & 11.7 & 31.8 & 19.2 \\
\hline \multirow{2}{*}{\multicolumn{18}{|c|}{ Predicted Operation }} \\
\hline & & & & & & & & & & & & & & & & & \\
\hline Run \#5, This calc - 2009 & $F$ & 85 & 70.9 & 161.1 & 317 & 21.1 & 56.9 & 96.7 & 65.6 & 61.4 & 34.1 & 35.3 & 13 & 13 & 12.5 & 32.8 & 19.3 \\
\hline Run \#6, This calc - 2009 & G & 85 & 70.9 & 161.1 & $\overline{317}$ & 40.8 & 56.9 & 96.7 & 85.1 & 81 & 53.7 & 54.9 & 13 & 13 & 12.5 & 32.8 & 19.3 \\
\hline
\end{tabular}

- Data not available

\section{Pressure Differential of Components}

KOP

Settler(s) (across bank)

Filter Vessel(s)
IXM(s)

\begin{tabular}{ccccccccc}
\multicolumn{8}{c}{ Special Conditions: } & \\
$\mathrm{A}$ & $\mathrm{B}^{1}$ & $\mathrm{C}^{2}$ & $\mathrm{D}^{3}$ & $\mathrm{E}^{4}$ & $\mathrm{~F}^{5}$ & $\mathrm{G}^{6}$ & \\
14 & 24 & 28 & 16 & 36 & 36 & 16 & $(\Delta \mathrm{p}(\mathrm{psi}) @ 320 \mathrm{gpm})$ \\
4 & Auto Calc & Auto Calc & Auto Calc & Auto Calc & Auto Calc & Auto Calc & $(\Delta \mathrm{p}(\mathrm{psi}) @ 320 \mathrm{gpm})$ \\
102 & 160 & 160 & 320 & 160 & 160 & 160 & $(\Delta \mathrm{p}(\mathrm{psi}) @ 320 \mathrm{gpm})$ \\
54 & 112 & 100 & 100 & 100 & 100 & 100 & $(\Delta \mathrm{p}(\mathrm{psi}) @ 320 \mathrm{gpm})$
\end{tabular}

1 Throttle added on P-3 inlet and IXM discharge.

$2 \mathrm{P}-3$ inlet and $\mathrm{P}-1$ and $\mathrm{P}-2$ discharge throttled. Slight increase in KOP vessel pressure differential.

3 P-3 inlet \& outlet throttled significantly, P-2 discharge throttled, FVs heavily loaded KOP lightly loaded.

4 No throttles on pumps. Added large throttle on $K O P$ inlet $(K=275)$ and increased $K O P$ pressure drop.

5 Same as 4 above except removed large throttle on KOP inlet.

6 Same as 5 above except KOP loss is matched to new KOP pressure loss data from condition $D$ above. 
Figure 2 depicts the pressure profile for model runs 3, 4, 5, and 6 shown in Table 1.

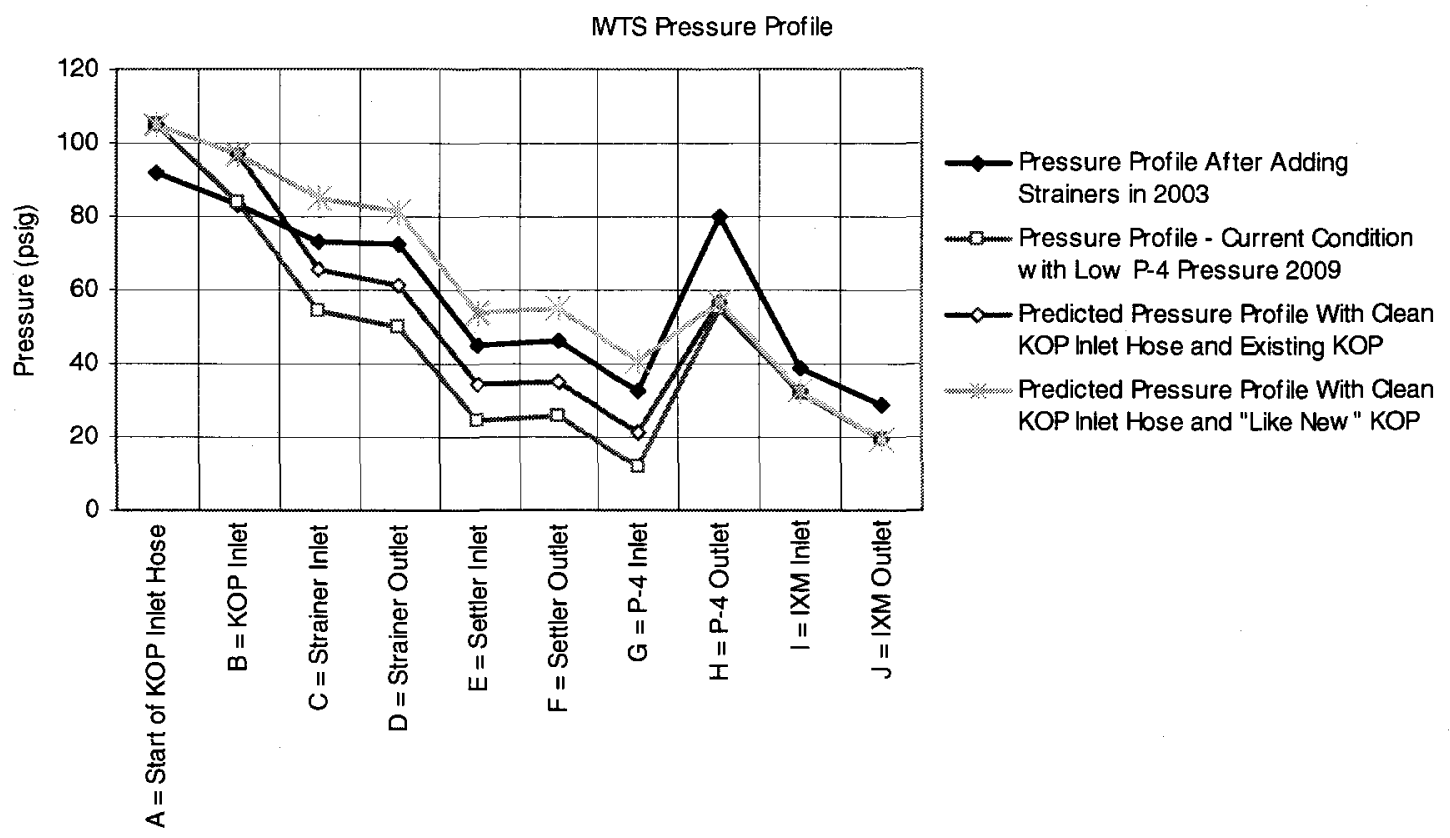

Figure 2 - IWTS Pressure Profile

The trace for "Pressure Profile after Adding Strainers in 2003" is the result of bench marking the hydraulic model against August 2003 field recorded values obtained shortly after the IWTS strainers downstream of the KOP were added.

The trace for "Pressure Profile - Current Condition with Low P-4 Inlet Pressure 2009" is a result of using the bench marked hydraulic model with additional losses included across the KOP Inlet hose (i.e., between points " $A$ " and "B") and the KOP vessel itself. Losses for these items were increased until the hydraulic model output matched current field recorded values.

The trace for "Predicted Pressure Profile with Clean KOP Inlet Hose and Existing KOP" is the hydraulic model's prediction of system pressures if the KOP inlet hose is cleaned or replaced but no changes are made to the existing KOP.

The trace for "Predicted Pressure Profile with Clean KOP Inlet Hose and "Like New" KOP" is the hydraulic model's prediction of system pressures if the KOP inlet hose and the KOP's inlet pipe are cleaned or replaced.

A slight discrepancy is shown in the pressure profile because the flow rates of P-1, P-2 and P-3 during the 2003 time frame were significantly different than those in use today. This discrepancy is attributed to more throttling applied to the inlet of pump P-3 with valve FRW-V-051 perhaps done to increase the flow from either P-1 or P-2.

Furthermore, the pressure differential recorded in the field for the filter vessels and IXMs for 2003 were much higher than current readings. This is attributed to the filter vessels requiring a backwash. 


\subsection{Field Evaluation}

Preliminary discussions of possible causes of low inlet pressure at the P4 pump included accumulation of material, leakage, or obstruction of the 3" (KOP to Settler) and 4" (Settlers to P4 Booster Pump) IWTS hoses. In response, the system was walked down and video taped and dose rates were taken along the hoses. In addition there was speculation that significant quantities of material were accumulating in the South Load Out Pit (SLOP) during backwash of the garnet filters. In response, observation was made of the SLOP.

\subsubsection{Walk Down of IWTS Hoses}

The IWTS hoses were walked down and video taped. No evidence of leakage or obstruction was observed. Even a small leak would be expected to produce a visible cloud or a disturbance of the floor sludge in the vicinity.

\subsubsection{Dose Rates of IWTS Hoses}

Dose rates were taken along the IWTS hoses. Most of the readings were less than $5 \mathrm{R} / \mathrm{hr}$. In three locations, readings were taken that were greater than 10 and as high as $60 \mathrm{R} / \mathrm{hr}$. In each of those cases other readings were taken of the floor in the same vicinity and the readings of the floor were higher than adjacent readings of the hoses. This indicates that the high readings are attributed to local background and do not suggest the accumulation of radioactive material in the hoses.

\subsubsection{South Load Out Pit}

Video taped observation of the SLOP only shows a trace of floor sludge, consistent with settling from a cloudy basin. There is no evidence in the SLOP that significant quantities of visible material are being discharged to the SLOP during garnet filter backwashing.

\subsection{Historical Issues}

Preliminary discussions of possible causes of IWTS symptoms included speculation on damage to or redesign of the IWTS hoses. Following is the history of the replacement of the IWTS hoses.

Oct 2000 - A new set of 3-inch (KOP to Settlers) and 4-inch (Settlers to P4 Booster Pump) hoses were installed to resolve an NCR on fitting pressure ratings.

Sep 2005 - A section of 4-inch hose was replaced in the east bay as a result of HIH construction or debris removal activity. The 75 foot section was replaced by 3 sections of 25 foot hose. This was done after completion of the fuel processing campaign which ended in October, 2004.

March 2008 - A complete set of 3-inch and 4-inch hoses were procured when IWTS was re-assembled after completion of the floor and pit sludge vacuuming in the west bay. Operations was able to re-use and connect the existing hoses. Spare hoses are currently stored at the 2101-M warehouse. 


\section{KBC-40653, Rev. 0}

Facility operators have reported that the IWTS hoses are heavy and difficult to move. There may be material accumulations that are not reflected in system performance nor dose readings. However, additional accumulations may impact system performance in the future and could call for the replacement of hoses with the spares. 
KBC-40653, Rev. 0

\section{Recommendations}

\subsection{Near Term}

Recent difficulties with low inlet pressure at the P4 booster pump are attributed to restrictions in the KOP inlet hose and the KOP itself. As soon as practical, the following actions should be completed.

- Replace the 2" KOP inlet hose. This hose will be manufactured and pressure tested offsite. A requisition for the replacement hose is in place. Modeling predicts that this change alone will increase the P4 inlet pressure from 11.7 to 21.2 psig.

- Use a borescope to observe the hard pipe inlet to the installed KOP.

- To the extent practical, remove any nested material in the KOP inlet.

\subsection{Long Term}

The following recommendations are offered to sustain IWTS performance during its planned continued use.

- Performance Evaluation - The hydraulic model of the IWTS proved valuable in diagnosing the current problem. Performance data from the IWTS should routinely be measured against the model to identify developing issues as early as possible.

A systems engineering evaluation of all systems to be used to support KOP Material disposition, including $\mathrm{K}$ Basins, transport systems, CVDF, and CSB, is recommended to identify and prioritize overall spare parts needs.

- Spare Parts Inventory -

Spare parts currently on hand include two (2) pumps that can replace $\mathrm{P} 1$ or $\mathrm{P} 2$, and a full set of 3" and 4" IWTS hoses.

Replacement pumps for P3 and P4 are currently being ordered.

A replacement for the KOP inlet hose is being ordered, but will be used as soon as possible. Another replacement hose should be ordered as a spare.

Spare parts needed to operate the PCM were also considered. PCM bearing cups are available in spare inventory. PCM locking pins (assemblies) are not available in spare inventory. These are manufactured components with long lead times. It is recommended that four complete assemblies (two of each configuration) be added to spare parts inventory.

- System Modification - Consideration could be given to removing the KOP from the system. The presence of the KOP strainers downstream of the KOP assures removal of material greater than 600 microns. However, removal of the KOP would require modification of the Safety Basis and could have potential impact on the acceptance (as sludge) of material accumulated downstream of the KOP. 
Consideration should be given to re-configuration of the front end pumps to allow actual pump performance (flow/dP) to be monitored. P1 and P3 are now only providing make-up flow to achieve the total design flowrate. It could be beneficial to increase the P2 pump flowrate to $100-150 \mathrm{gpm}$ from the current operating range of $60-80 \mathrm{gpm}$. 


$$
\text { KBC-40653, Rev. } 0
$$

\section{References}

System Design Description for the KW Basin Integrated Water Treatment System, SNF4449, Rev. 0, April, 2000

H-1-80550, KW Fuel Storage Basin P\&ID, U.S. Department of Energy

H-1-83301, KW Fuel Storage Basin IWTS PFD, U.S. Department of Energy 